\title{
Quantitative modeling of fuel retention in the JET-C and JET-ILW wall configurations by WallDYN and predictions for ITER
}

\author{
K. Schmid ${ }^{*, a}$ K. Krieger ${ }^{\text {a }}$ S. W. Lisgo ${ }^{b}$ G. Meisl a \\ S. Brezinsek ${ }^{c}$ JET EFDA Contributors ${ }^{d}$ \\ ${ }^{a}$ Max-Planck-Institut für Plasmaphysik, Boltzmannstraße 2, D-85748 Garching b. \\ München Germany \\ ${ }^{\mathrm{b}}$ ITER Organization, Route de Vinon sur Verdon, 13115 Saint Paul-lez-Durance, \\ France \\ ${ }^{\mathrm{c}}$ Assoc. EURATOM FZJ, Institute for Energy $\&$ Climate Research, Jülich, \\ Germany \\ ${ }^{\mathrm{d}}$ See the Appendix of F. Romanelli et al., Proceedings of the 24th IAEA Fusion \\ Energy Conference 2012, San Diego
}

\begin{abstract}
The fuel retention in ITER will be dominated by co-deposition. Therefore predictions of retention in ITER require a global model of migration of light (Be, C) impurities and their co-deposition with hydrogenic species. To track the global material erosion/deposition balance and the resulting formation of mixed material layers the WallDYN code has been developed. This paper describes the application of WallDYN to the interpretation of results on deposition and co-deposition in JET experiments both in the ITER like wall (ILW) and full carbon configuration. The calculations show qualitative agreement with the Be deposition patterns determined from post-campaign wall tile analysis. The calculated retention results for $\mathrm{C}$ and $\mathrm{Be}$ first wall configurations even show quantitative agreement with experimental gas balance measurements when long term outgassing is taken into account. Applying WallDYN to ITER for different first wall and plasma configurations shows that for the current $(\mathrm{Be}+\mathrm{W}$ only) material choice, retention will not limit ITER operation whereas $\mathrm{C}$ would increase retention by factors 10 to 100 , leading to unacceptably high fuel retention by co-deposition.
\end{abstract}

Key words:

PACS: 61.80.Jh, 52.25.Vy, 52.65, 52.40.Hf

PSI Keywords: DIVIMP, Erosion \& Deposition, Sputtering, Edge modeling 


\section{Introduction}

The migration of first wall material due to erosion, plasma transport and deposition is one of the key challenges in current and future fusion devices. It affects the lifetime of wall components in net erosion regions and can lead to formation of material layers and strong fuel retention via co-deposition in net deposition regions of the first wall [1]. To predict erosion/deposition patterns and to understand the underlying principal processes, a global simulation code is required that couples the evolution of the first wall surface composition to plasma impurity transport. To that end, the WallDYN $[2,3]$ code was developed. It maintains a strict global material balance of all eroded and deposited material and allows to track the chain of erosion/re-deposition/re-erosion and re-deposition steps that define where material is finally net-deposited or net-eroded. WallDYN couples the output from state of the art models (e.g. SDTRIM.SP $[4,5]$ ) for the surface processes (e.g. erosion, reflection, sublimation) with material redistribution data from trace impurity plasma transport models (e.g. DIVIMP [6]) in a self-consistent simulation. For each plasma exposed location on the discretized first wall contour it calculates the time evolution of both the concentration of elements in the surface and in the incident particle flux ( $\equiv$ particles per area $\times$ time). WallDYN also derives the growth rate of depositing layers, which can be converted to a fuel retention rate via co-deposition by multiplying it with the hydrogen concentration in the layer, which is taken from scaling laws fitted to lab experiments [7-9]. To benchmark the WallDYN model, it was applied to the JET ITER-Like Wall experiment (JET-ILW) [10], which mimics the ITER first wall material configuration and is thus an ideal environment to validate the predictive significance of the WallDYN deposition/retention calculations for ITER.

The WallDYN simulations were performed for ohmic and H-mode plasma scenarios used in global retention studies both during the JET-ILW campaigns and previous JET configuration with CFC walls (JET-C) campaigns. This allowed quantitative benchmarking of the code predictions against the strong difference in global fuel retention between C- and Be-dominated JET configurations. In addition to the diverted plasma phases, limiter plasma config-

\footnotetext{
* K. Schmid Email address: klaus.schmid@ipp.mpg.de (K. Schmid).
} 
urations were also modeled to include the influence of plasma ramp-up and ramp-down on Be deposition as proposed in [11].

The calculated poloidal erosion/deposition patterns are compared to experimentally determined erosion/deposition distributions in $[12,13]$. From the calculated layer growth the co-deposition based retention rates were derived using the scaling laws from [7-9]. These retention rates are then compared to the results from gas balance measurements during JET-ILW and JET-C configurations taken from [14].

Applying the same model as for the JET calculations, the impurity migration and resulting fuel species co-deposition in ITER for different wall configurations and background plasmas was calculated. The background plasmas span a large range of plasma parameters at the wall (temperatures, densities, and fluxes) and scrape-off layer (SOL) impurity migration parameters (SOL flows, screening). In the WallDYN calculations based on these background plasmas different first wall material configurations were then compared with respect to the fuel retention rate by co-deposition.

The paper will first given a short introduction into the WallDYN model including the implemented improvements since the original WallDYN publication [2]. Then the JET calculations results will be presented together with a comparison to the corresponding experimental data. Finally the WallDYN prediction on co-deposition in ITER will be presented.

\section{Model Description}

\subsection{Basic WallDYN concepts}

The WallDYN model is described in detail in [2] therefore only a short summary of the main changes since the original publication are given here.

The first noteworthy improvement is that the incident particle spectrum now is charge state resolved. This removes the necessity of flux averaged species energies described in [2]. In the current WallDYN version each species in the incident spectrum impinges on the surface with an energy determined by the local sheath and the Bohm velocity according to eq. 1. 


$$
\begin{aligned}
E_{e j, w k} & =3 q j T_{w k}^{e}+2 T_{w k}^{I} \\
q j & =\text { Charge state in which element ej hits wall element wk }
\end{aligned}
$$

Compared to the equations in [2] this added flux resolution requires additional sums over all charges states in several of WallDYN's underlying equations to determine various fluxes. The charge resolved versions of the most important equations are summarized in eq. 2 .

$$
\begin{aligned}
\Gamma_{e i, w s}^{\text {Ero }} & =\text { Erosion flux of component ei from wall element ws } \\
& =\sum_{e j=0}^{\mathrm{NElem}} C_{e i, w s}\left(\sum_{q j=0}^{\mathrm{NChrg} \mathrm{ej}} \Gamma_{e j, q j, w s}^{\mathrm{In}} * Y_{e i, e j}\left(E_{q j, w s}, \delta_{e k \ldots N}\right)\right)
\end{aligned}
$$

$Y_{e i, e j}\left(E_{q j, w s}, \delta_{e k \ldots N}\right)=$ Sputter yield of element ei by element ej on wall element ws as function of energy $\mathrm{E}$ and composition $\delta$

$C_{e i, w s}=$ Concentration of element ei on wall tile ws

$\Gamma_{e i, w s}^{\text {Refl }}=$ Reflected flux of component ei from wall element ws

$$
=\sum_{q i=0}^{\mathrm{NChrg}_{\mathrm{ei}}} \Gamma_{e i, q i, w s}^{\mathrm{In}} * R_{e i, w s}\left(E_{q i, w s}, \delta_{e j \ldots N}\right)
$$

$$
\begin{aligned}
R_{e i}\left(E_{q i, w r}, \delta_{e j \ldots N}\right)= & \text { Reflection yield of element ei on wall tile wr } \\
& \text { as function of energy E and composition } \delta \\
\Gamma_{e i, q i, w r}^{\mathrm{In}}= & \text { Linear equation for evolution of flux } \\
& \text { of element ei, charge state qi on wall element wr } \\
= & \sum_{w s=1}^{\text {NElem }}\left(\Gamma_{e i, w s}^{\text {Ero }}\left(\Gamma_{e j, q j, w s}^{\mathrm{In}}\right)+\Gamma_{e i, w s}^{\mathrm{Ref}}\left(\Gamma_{e i, q i, w s}^{\mathrm{In}}\right)\right) * \xi_{e i, q i, w s, w r} \\
\xi_{e i, q i, w s, w r}= & \text { Charge state resolved redistribution matrix } \\
= & \text { Fraction of element ei } \\
& \text { emitted from ws ending up on wr at charge state qi }
\end{aligned}
$$

The second noteworthy improvement is the implementation of a virtual diagnostic. Given a poloidal distribution of impurity influx into the plasma it calculates the corresponding impurity densities on the calculation grid. From that, applying line integration and using the appropriate $\left(T_{e}, n_{e}\right.$ from plasma background) ADAS [15] data, it calculates the spectroscopic signals $\left(P h /\left(\mathrm{cm}^{2} s\right)\right)$ for a given line of sight (LOS) that can be directly compared to experimental data. The charge state resolved impurity density on the grid is calculated by multiplying the impurity influx vector with a feedback matrix $\Omega_{e i, w k, q i, i k, i r}$ which describes the contribution of the impurity influx of element ei from 
wall element wk to the density of ei at charge state qi $\left(m^{-3}\right)$ at calculation grid cell (ik, ir). $\Omega_{e i, w k, q i, i k, i r}$ is determined during the calculation (see [2]) of the redistribution matrix $\xi_{e i, q i, w s, w r}$ (see eq. 2): For a given impurity influx $\Gamma_{e i, w k}$ of element ei from wall element wk the charge state resolved impurity density $\rho_{e i, w k, q i, i k, i r}$ in each grid cell is stored. From $\rho_{e i, w k, q i, i k, i r} \Omega_{e i, w k, q i, i k, i r}$ is determined by normalizing with $\Gamma_{e i, w k}$.

\subsection{Plasma impurity transport}

To determine the redistribution matrix $\xi_{e i, q i, w s, w r}$ of the impurity migration WallDYN relies on DIVIMP [6]. DIVIMP is a 2D Monte-Carlo code that calculates the transport of impurities in the plasma in trace approximation on a static background plasma. For the JET calculations the background plasma was calculated using a quasi 1D "onion-skin" plasma model (OSM) [16,17] on a computational grid. While the onion-skin model only solves a simplified version of the Braginskii equations, it allows a very close match to the experimentally observed plasma parameters because measurements of temperature and density at the divertor plates and the outer mid-plane are used as boundary conditions.

For the ITER calculations the approach is slightly different: The near SOL plasma is taken from a SOLPS [18] calculation, while the far SOL plasma is based on a simple isothermal, purely convective plasma model that prescribes plasma parameters that are consistent with observed experimental trends [17]. The differences in the ITER background plasmas can be characterized by several parameters which are summarized in table 1. All plasma have H-mode pedestals but differ in the following parameters: "Sep. distance" is the distance between the primary and secondary separatrix at the outer mid-plane. "Density" is the density close to the wall in the main chamber. "Far SOL $T_{e} / T_{i}$ " is the ratio of $T_{e}$ to the $T_{i}$ of the majority species (Deuterium). "Far SOL $V_{\text {Perp }}$ " is the far SOL convection velocity. "Far SOL T-grad" is an option that switches from an isothermal far SOL to far sol with a $T_{e}$ gradient. "Near SOL flow" enforces a parallel flow in the background plasma. The so defined background plasmas (see also [17]) span a large range of wall conditions in terms of flux and particle energy. For the ITER cases only the D and D-CX fluxes were used as constant background fluxes in WallDYN although 
the SOLPS part of the background plasmas included seeded impurities like Ne to cool the divertor plasmas. To test the influence of Ne it was introduced into the some test calculations but the effect was small: The Be erosion and thus also the $\mathrm{Be}$ or $\mathrm{C}$ co-deposition rate only increased by $\approx 20 \%$ thus the conclusion drawn from the pure D plasma backgrounds are not affected by seeded impurities like Ne.

For both JET and ITER all the background plasmas are defined on a computational grid that tightly matches the entire first wall without gaps. This allows to directly extract wall fluxes around the entire poloidal circumference without the need the extrapolate plasma fluxes across gaps between the calculation grid and the wall. Since DIVIMP is a 2D transport code the wall is assumed to be toroidally symmetric i.e. for JET the guard limiters are approximated as belt limiters. (See also section 3.1.1) In the DIVIMP calculations the perpendicular transport of impurities is controlled by the perpendicular diffusion coefficient

$D_{\text {Perp }}\left(\mathrm{m}^{2} \mathrm{~s}^{-1}\right)$. In the calculations presented here $D_{\text {Perp }}=1.0 \mathrm{~m}^{2} \mathrm{~s}^{-1}$ was used unless stated otherwise.

The focus in this paper is to investigate the impurity migration, therefore background plasma parameters close to experimentally observed values is required. Thus in an effort to make the solution consistent with the generally observed experiment trend, background plasma flows were imposed on the OSM solution. The stagnation point is located close to the outer mid-plane and the parallel flow velocities are $\mathrm{M} \approx 0.5$ on the HFS.

\subsection{Background impurity fluxes}

WallDYN distinguishes between dynamic impurity fluxes (e.g. Be, C, W) due to wall erosion and migration and static particle fluxes (e.g. D, D-Charge exchange (CX), O)from the background plasma that are constant in time. The OSM model only yields a pure D background plasma. To include the effect of additional erosion by impurities resulting from the ionization of the residual gas, O was added to the WallDYN calculations for JET. O was chosen since an air leak was present during the first JET-ILW campaign plasmas that are modeled in this paper. To add a realistic, charge state resolved background flux of $\mathrm{O}$ a poloidally uniform $\mathrm{O}$ influx $\Gamma_{O}$ is assumed. Using an $\mathrm{O}$ feedback matrix the spectroscopic line intensities in the JET main chamber were cal- 
culated for a given $\Gamma_{O}$. Then $\Gamma_{O}$ was adjusted until the calculated, match the experimentally observed spectroscopic line intensities. An O II line at $441.9 \mathrm{~nm}$ viewed through a horizontal main chamber LOS (KSRc track 12) was used for the comparison.

Based on this $\Gamma_{O}^{\text {Match }}$ the charge state resolved $\mathrm{O}$ impurity flux at each poloidal location was determined using the appropriate O-redistribution matrix $\xi_{e i, q i, w s, w r}$. This resulted in $\mathrm{O}$ flux fraction $\leq 1 \%$ throughout the entire poloidal circumference and $\mathrm{O}$ impurity concentrations ranging from 0.1 to $0.5 \%$ in the divertor and up to $1 \%$ in the main chamber plasma.

Due to the low flux fraction $\mathrm{O}$ is only treated as a constant background species and no formation of $\mathrm{O}$ containing mixed layers was included in the simulation. This can be justified by the high re-erosion rate by $\mathrm{D}$ for which no formation of $\mathrm{BeO}$ during the discharge is expected. Therefore the $\mathrm{BeO}$ formation is not included in the WallDYN calculations and $\mathrm{O}$ only acts as an additional eroding species.

It should be noted here that any effect of impurities on the background plasma is already intrinsically included in the plasma solution since it is based on experimental $T_{e}$ and $n_{e}$ values. This of course only holds as long as the plasma impurity concentration do not exceed the experimentally observed values.

\begin{tabular}{lll}
\hline Parameter & Range & "Affects" \\
\hline Sep. distance & $10 \mathrm{~cm}$ vs $4 \mathrm{~cm}$ & Charge states at the wall \\
Density & Low/Medium/High & Wall fluxes \\
Far SOL $T_{e} / T_{i}(\mathrm{eV})$ & $T_{e}=10 T_{i}=20 \mathrm{vs.}$ & Erosion rates \\
& $T_{e}=20 T_{i}=40$ & \\
& & \\
Far SOL $V_{\text {Perp }}$ & $35 \mathrm{~m} / \mathrm{s}$ vs. $100 \mathrm{~m} / \mathrm{s}$ & $\begin{array}{l}\text { Far SOL density } \\
\text { (impurity screening) }\end{array}$ \\
& & \\
Far SOL T-grad & Off vs. On & Erosion \\
Near SOL flow & 0 vs. 0.5 Mach & Transport to \\
& & inner divertor \\
\hline
\end{tabular}

Table 1

Parameter differences in the ITER background plasmas and how they qualitatively "affect" the WallDYN solution. 


\subsection{Calculation of retention by co-deposition}

From the net deposition rates $\eta_{e i, w k}\left(m^{-2} s^{-1}\right)$ of element ei on wall element wk calculated by WallDYN the retention via co-deposition is determined by multiplying $\eta_{e i, w k}$ with the $D / X_{e i, w k}$ ratio. The $\mathrm{D} / \mathrm{X}$ ratio describes the fraction of $\mathrm{D}$ per host atom $\mathrm{X}$ in the layer given the local (i.e. on wall element wk) parameters like D particle energy, D flux and temperature. The D/X ratios used here are taken from [7] for Be, from [8] for $\mathrm{C}$ and from [9] for $\mathrm{W}$ co-deposits.

The scaling laws are only valid for a limited range of energies, temperatures and fluxes. For both the JET and ITER simulations these parameter ranges are exceeded at certain wall locations. Since scaling laws must not be extrapolated the input parameters are clamped to the validity range of the scaling laws.

\subsection{Modeled JET shots}

As part of the WallDYN JET benchmark background plasmas corresponding to two JET-ILW shots were modeled:

- 80295 an ohmic shot that was used during the Be migration studies [11] during initial start up phase of JET with ILW wall.

- 83559 an H-Mode shot also taken from the first JET-ILW campaign.

While both plasma are from the JET-ILW campaign they are similar to plasmas from the JET-C campaigns and can therefore be used to compare retention between $\mathrm{C}$ and Be dominated wall configurations.

The background plasma for the diverted flat top phase of 80295 has already been used in [11] to interpret the Be migration studies. In [11] the spectroscopic Be signals could not be explained based on the diverted phase of 80295 alone. It was suggested that the extended limiter phase prior to the first diverted plasma and during the ramp up phase of each shot should be taken into account. Therefore also a limiter plasma, corresponding to the magnetic equilibrium (see Fig. 1) at 6sec into shot 80295, was created using the OSM approach described in section 2.2. The radial profiles of temperature and den- 
sity used in the OSM are taken from [19]. The OSM boundary conditions are defined along the radial line in Fig. 1 where $T_{e}$ and $N_{e}$ decay linearly from 30 to $15 \mathrm{eV}$ and $5.0 \times 10^{18}$ to $0.5 \times 10^{18}\left(\mathrm{~m}^{-3}\right)$ respectively. The limited plasma is in contact with both inner and outer wall limiters with the last closed flux surface (LCFS) is touching the outer limiter. This limiter plasma is representative only for the limiter phases during the JET-ILW start up for Be migration experiment described in [11]. Later in the campaign the limiter plasmas had higher densities and thus less impact energy and Be sputtering. For an overview of the JET-ILW limiter plasmas see [20].

The background plasma for the H-mode shot 83559 was also created using the OSM approach using inter ELM profiles of $T_{e}$ and $N_{e}$ at the targets and upstream at the outer mid-plane. The conditions for shot 83559 are also described in [20]

For the JET-C cases a pure C wall and for JET-ILW a pure Be main wall was assumed as initial conditions i.e. no impurities in the wall prior to plasma exposure. According to [20] this is a reasonable assumption for JET-ILW. For JET-C this amounts to shots long after the last Be evaporation, as the ones used for gas balance measurements in [14].

\section{Results and Discussion}

\subsection{WallDYN modeling of JET results}

The aim in the JET modeling work was to validate the WallDYN model both by comparing it to global (e.g. gas balance) and local (e.g. deposition patterns) experimental parameters. Three WallDYN cases were modeled that yielded data directly comparable to the experiment.

\subsubsection{Be erosion deposition patterns}

To determine the equilibrium Be erosion deposition patterns WallDYN runs were performed based on the diverted background plasmas for shot 80295 and 83559. In the H-mode case the wall flux is in the range of $1.0 \times 10^{20} \mathrm{~m}^{-2} \mathrm{~s}^{-1}$ at the wall and $1.0 \times 10^{23} \mathrm{~m}^{-2} \mathrm{~s}^{-1}$ at the strike points. The fluxes in the ohmic 
case are roughly an order of magnitude lower. The electron temperature at the outer strike point peaks at $33 \mathrm{eV}$ for the ohmic and $20 \mathrm{eV}$ for the $\mathrm{H}$-mode case resulting in $\mathrm{W}$ sputtering by impurities only in the simulation in both cases.

In the calculations the evolution of the impurity flux and surface composition was followed until they became constant i.e. equilibrium was reached. For all wall elements to equilibrate it typically took $\approx 400 \mathrm{sec}$ of constant ohmic (80295) or H-mode (83559) plasma exposure. It should be noted that not all wall elements reach their equilibrium state equally fast: The higher the local wall flux the faster equilibrium was reached. The final $\eta_{e i, w k}=\frac{\partial \delta_{e i, w k}}{\partial t}$ corresponds to the net erosion $\left(\eta_{e i, w k}<0\right)$ or net deposition $\left(\eta_{e i, w k}>0\right)$ rate. In Fig. 2 the poloidal distribution of net erosion / deposition rates and Be surface concentrations are shown for the JET-ILW for the ohmic case 80295 in the presence of a background $\mathrm{O}$ impurity flux. The main wall is a net Be erosion zone except for some small areas. There is generally no net Be deposition on $\mathrm{W}$ divertor targets but a finite Be surface concentration of up to $20 \%$. There is minor Be deposition on the lower part of the inner and outer vertical targets and strong Be deposition on top of tile 1 "apron" (see Fig. 1 in [13]). This principal pattern is the same for all background plasma configurations. The absolute deposition rates depend on the presence of additional impurities (the added $\mathrm{O}$ influx described in section 2.3) and on the background plasma type (ohmic, H-mode). Also varying $D_{\text {Perp }}$ in the ohmic case from $1.0 \mathrm{~m}^{2} \mathrm{~s}^{-1}$ to $10.0 \mathrm{~m}^{2} \mathrm{~s}^{-1}$ decreases the Be deposition rate on top of tile 1 "apron" by a factor of $\approx 2$. The reason for this reduction is that a higher $D_{P e r p}$ results in less long range transport of $\mathrm{Be}$ and thus reduces the amount of eroded Be, eroded at locations far away, that ends up on top of tile 1.

For the ohmic and H-mode case, the Be deposition rate on top of tile 1 "apron" ranges from $0.4 \times 10^{20} \times 10^{20}\left(\mathrm{~m}^{-2} s-1\right)$ to $2.8 \times 10^{20} \times 10^{20}\left(\mathrm{~m}^{-2} \mathrm{~s}-1\right)$ depending on modeling assumptions and location.

The fact that H-mode and ohmic have similar Be deposition rates means that the higher Be influx in H-mode in canceled by a correspondingly higher Be erosion rate.

The addition of the O-impurity flux leads to a higher Be source in the main chamber and thus increases the Be deposition rate by a factor of $\approx 2$ in ohmic and only $\approx 1.3$ in $\mathrm{H}$-mode case. The smaller impact in the H-mode case is due to a lower, average $\mathrm{O}$ flux fraction. 
The location of the net $\mathrm{W}$ erosion zones are the same in ohmic and H-mode but differ in magnitude. The main areas of $W$ erosion are on the vertical part of tile 1 well below the apron, at the outer strike point on tile 5 and on the outer baffle and vertical target. The highest $\mathrm{W}$ net erosion rate is found at the outer strike point for the H-mode plasma with O-impurity flux with a value of $0.1 \times 10^{20} \mathrm{~m}^{-2} \mathrm{~s}^{-1}$. Without O-impurity flux this maximum value is reduced approximately by a factor of two. In reality the $\mathrm{W}$ erosion will be dominated by ELM activity in the H-mode case, therefore this calculated value is only a lower bound.

Comparing the distribution of Be net deposition and erosion with experimental data from $[12,13]$ shows good qualitative agreement: Be Deposition dominantly on tile one apron and little Be on the rest of the $\mathrm{W}$ divertor area. The actual quantitative numbers on Be layer growth on the tile 1 apron region are the cumulative result of erosion \& deposition during many different plasma scenarios. Assuming that shots modeled are a reasonable proxy for the majority of the JET-ILW plasmas, the experimentally determined layer thickness can be compared to the WallDYN net deposition rates. According to [12] the total divertor time in the initial JET-ILW campaign was $\approx 13 \mathrm{~h}$ and a Be layer thickness of 10 to $15 \pm 5 \mu \mathrm{m}$ which for a Be density of $\approx 1.0 \times 10^{29} \mathrm{~m}^{-3}$ results in a Be deposition rate of 2 to $3 \pm 1 \times 10^{19}\left(\mathrm{~m}^{-2} \mathrm{~s}-1\right)$. Compared to the calculated Be deposition rate on tile 1 apron this is lower by factor 3 to 10 . Which is not unreasonable since not all of the $13 \mathrm{~h}$ of divertor time were during steady state plasmas.

In the main chamber the comparison between WallDYN and the experiment is not straight forward due to presence of limiter shadow areas. Experimentally $[12,13]$ erosion is found on the apex of the limiters and some Be deposition in the limiter shadow. As WallDYN uses a 2D impurity transport code (DIVIMP) it can not handle limiter shadows. But since the total particle rate $\psi\left(s^{-1}\right)$ to the wall has to be the same, both in WallDYN ( $\psi$ distributed over entire wall) and the experiment ( $\psi$ focussed onto the limiters) the net change in Be: Be deposition in shadowed areas minus Be erosion at the apex, should be the same (except for non linearities due to Be self-sputtering). Therefore as the amount of Be deposition the divertor also shows a reasonable, quantitative match this net change in Be in the main chamber (三 the source of Be depositing in the divetor) must also be reasonably well described by WallDYN. 


\subsubsection{Influence of limiter phases on Be migration}

In [11] the observed time evolution of the Be II spectroscopy signals could not be explained based on the diverted phase of the discharge. It was speculated then, that the Be erosion and migration during the limiter phase may affect the Be II signal during the subsequent diverted plasma phase.

To investigate the influence of limiter phases the JET-ILW start up was modeled by a sequence of WallDYN calculations. The calculations were connected into a sequence by passing the final surface composition state of a particular calculation as the initial state of the following calculation, thus modeling the time evolution of the system under different plasma conditions. The sequence started with a long (540 sec.) limiter discharge mimicking the conditioning phase during the JET-ILW start up. This long limiter phase was followed by alternating limiter ramp up (12 sec.) and divertor (18 sec.) plasmas.

During the limiter phase the Be limiters are heavily eroded, mainly by Be selfsputtering. The eroded Be is transported to the divertor. For instance during the long 540 sec. limiter phase, a Be surface concentration of 10 to $20 \%$ is reached but no net Be layer growth occurs. The transport to the divertor only occurs via neutral Be emanating from the lower parts of the main chamber limiters. Any sputtered Be that is ionized, is guided by the magnetic field and ends up elsewhere in the main chamber. Of the total Be eroded during the limiter phase only $20 \%$ reach the divertor. The high field side (HFS) contributes $30 \%$ to the main chamber Be II spectroscopic intensities along the line of sight whereas the low field side (LFS) contributes 70\%. (See [11] for details of LOS). This asymmetry is due to the fact that the in limiter equilibrium used (see Fig. 1) the LCFS touches the LFS resulting in a stronger Be source at the LFS side.

In Fig. 3 the inner and outer divertor spectroscopic Be II signals are shown for the same wave lengths as in [11]. For each sequence the signal level at the beginning (i.e. directly after the limiter phase) and at the end of the divertor plasma phase are shown. The lines connect the symbols in temporal sequence. The Be II signal intensity is higher at the beginning and decreases significantly during the divertor plasma phase. This variation is due to the deposition of Be in the divertor area during the limiter phase which is subsequently eroded during the divertor phase. This erosion of Be results in a decrease in the Be II signal. During the next limiter phase the Be surface concentration is again 
replenished restoring the Be II signals at the beginning of the following divertor plasma phase.

In the experiment (plot 4. in [11]) the Be II signal varies by 20 to $30 \%$ during the divertor plasma phase, this compares well to the calculated variation of 40 $\%$ in the outer divertor and $20 \%$ in the inner divertor. Also shown in Fig. 3 is the range of experimentally observed absolute values of the Be II signal intensity (dashed and dotted lines). The calculated values match this range well, suggesting that both the background plasma and the calculated Be migration match the experimental data well.

While these plasma sequence calculations well explain the Be II signal variation during the divertor phase they do not match the long term, multi-shot trends in the Be II signal intensities seen in the experiments in [11]. To match these observed trends either more Be needs to be transported to the divertor during the limiter phase (which would then take longer to erode) or the main chamber Be source needs to vary. Therefore these discrepancies could be due to the fact that the 2D neutral transport model in DIVIMP underestimates the total amount of Be transported to the divertor or that the main chamber Be source varies due to a varying impurity content during the JET-ILW startup phase. Both possibilities will be explored in the future.

\subsubsection{Fuel retention in JET-ILW vs. JET-C}

The fuel retention via co-deposition depends on the total amount of eroded material (Be for JET-ILW and C for JET-C) that is subsequently deposited at some poloidal location and the $\mathrm{D} / \mathrm{X}(\mathrm{X}=\mathrm{C}$ or $\mathrm{Be})$ ratio at that location. The $\mathrm{D} / \mathrm{X}$ ratio (see section 2.4) depends on temperature, D energy and the layer growth rate or the flux ratio of the depositing species to the fuel species. Apart from temperature all the other parameters are readily available during a WallDYN calculation. The temperatures are taken from experiment (according to [14]) as follows: For the ohmic case (shot 80295) the entire wall is assumed to be at base temperature of $473 \mathrm{~K}$. For the H-mode case the outer limiters are at $550 \mathrm{~K}$, the outer strike point at $1400 \mathrm{~K}$, the inner strike point at $800 \mathrm{~K}$ and the inner limiters at $600 \mathrm{~K}$. Since most of the areas where co-deposition occurs are far away from these high temperature regions, the choice of these temperatures has only minor effect on the calculated retention. The resulting $\mathrm{D} / \mathrm{X}$ ratios for $\mathrm{Be}$ and $\mathrm{C}$ are quite similar (on average 0.01 to 0.4 ) which is in line 
with [13]. What is different between $\mathrm{Be}$ and $\mathrm{C}$ is that in the divertor, region where most of the deposition takes place, $\mathrm{D} / \mathrm{Be}$ is much less than $\mathrm{D} / \mathrm{C}$. This is one reason for less retention by co-deposition in the JET-ILW vs JET-C.

To compare the calculated retention rates $(D / s)$ to the experimental gas balance data in [14] the effect of long term outgassing has to be taken into account. The reason is that the scaling laws to derive the $\mathrm{D} / \mathrm{X}$ ratios were determined from post mortem analysis of co-deposited layers and thus intrinsically lack the long term outgassed fraction of the retained fuel species. For JET-ILW the outgassing corrected data $(\approx 1.5 \mathrm{~h}$ of outgassing) was readily available from [14]. For C, according to the data in [21] the ratio of gas-balance to post mortem is $\approx 5$.

Using the ohmic (80295 flat top diverted phase) and H-mode (83559 flat top diverted phase) plasma configuration the $\mathrm{D}$ retention rate by co-deposition in equilibrium was calculated with and without the presence of $\mathrm{O}$ impurities. These calculations were performed both for the JET-ILW and JET-C wall material configuration. The resulting retention rates are compared to the experimental data from [14] in Fig. 4. The calculation for ohmic and H-mode match the experimental data quite well where long term outgassing is included. As in the experiment the retention in ohmic and H-mode plasmas is roughly a factor 10 lower for the JET-ILW compared to the JET-C material configuration.

Since for the ohmic plasma case adding $\mathrm{O}$ as impurity made a significant difference in the Be deposition rates (see section 3.1.1) the corresponding retention rate is also shown in Fig. 4. It actually matches the experimental value better than the D only plasma background case.

\subsection{Fuel retention predictions for ITER}

The comparison of WallDYN with the experimental results on Be deposition and co-deposition from the JET-ILW and JET-C campaigns has demonstrated its predictive capabilities. Therefore it seems justified to apply the same model to predict retention by co-deposition for ITER. Using the ITER background plasma cases described in section 2.2, WallDYN calculations for different first wall material configurations were compared: A full Be first wall and $\mathrm{W}$ divertor with CFC strike point areas = CASEA, a full Be first wall and a full $\mathrm{W}$ 
divertor $=$ CASEB and full $\mathrm{C}$ first wall and divertor $=$ CASEC . The result of this extrapolation is summarized in Fig. 5 which shows the experimental (from [14]) and calculated JET-ILW and JET-C values together with the extrapolation to ITER.

The simulations showed that CASEB has the lowest retention due to codeposition whereas CASEA and CASEC feature on average a 10 and 100 times higher retention rate respectively. For case C only 100 to 700 full 400 second ITER discharges would be possible before hitting the T-limit of $700 \mathrm{~g}$ whereas for CASEB between 3000 and 20000 full 400 second ITER discharges are possible depending on the plasma configuration. These numbers are in line with previous estimates in [1] based on a simple first wall flux scaling. However independent on the wall material configuration, the different background plasmas result in a factor $\approx 10$ variation in co-deposition despite similar total wall fluxes. These strong variations show that a simple wall flux scaling is not enough for predicting retention in ITER for various plasma conditions. Still, for the current ITER material choice (CASEB) fuel retention by co-deposition will not limit the ITER operation.

\section{Summary}

The global impurity migration code WallDYN is compared to experimental data on Be migration and co-deposition in both JET-ILW and JET-C material configurations.

The calculations qualitatively reproduce the Be deposition patterns from the JET-ILW campaign: Strong deposition on tile 1 "apron" and little to no deposition on the rest of the $\mathrm{W}$ divertor. A coarse quantitative comparison of the calculated Be deposition on tile 1 "apron" is within a factor of 3 to 10 of the experimentally measured values.

WallDYN calculations have shown that the limiter discharges in between diverted plasma phases have a strong impact on Be migration studies. The strong erosion of Be during the limiter phase (dominated by Be self-sputtering) leads to deposition of significant amounts of Be in the divertor region which affects the spectroscopically measured Be line intensities during the flat top divertor phase of the discharge.

A comparison of the calculated retention rate by co-deposition with exper- 
imental data from gas balance measurements for the JET-ILW and JET-C configuration shows that both, WallDYN and experiment, yield a factor 10 lower retention for Be compared to $\mathrm{C}$ wall components. When long term outgassing is taken into account WallDYN can even quantitatively match the experimental retention rate values for ohmic and H-mode plasmas.

Applying the same process physics as for the JET-ILW calculations WallDYN was used to predict co-deposition for ITER, for a wide range of ITER plasmas conditions. These calculations showed that $\mathrm{C}$ leads to unacceptably high retention rates whereas the current ITER material choice (Be main wall and full $\mathrm{W}$ divertor) will not limit ITER operation. 


\section{Figure \& Table captions}

Fig. 1

DIVIMP limiter and divertor phase grid for 80295. The red line marks the location where the OSM input radial $T_{e}$ and $N_{e}$ profiles are specified. Also shown are the boundary markers of wall regions (short lines perpendicular to first wall).

Fig. 2

The Be net erosion / deposition rates and Be surface concentrations for JETILW shot 80295 (ohmic) with O-impurity flux vs. WallDYN wall index along the entire poloidal circumference, starting with index $=1$ at the lower end of the inner wall limiters. The vertical lines mark the wall index where a particular section of the first wall starts. They match the markers in Fig. 1.

Fig. 3

The calculate varition in the Be II signal in the inner and outer divertor during the sequence of limiter and divertor plasmas. Also shown is the range of the experimentally observed values.

Fig. 4

Comparison of WallDYN calculated retention rates with experimental results for different plasma configurations. The red arrows denote experimental and calculated values that can be compared directly. 


\section{Figures}

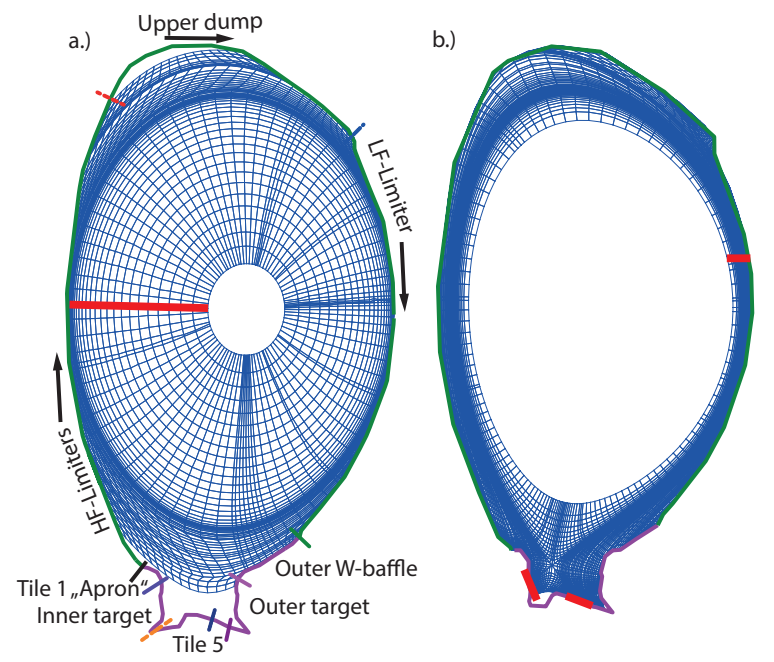

Fig. 1.

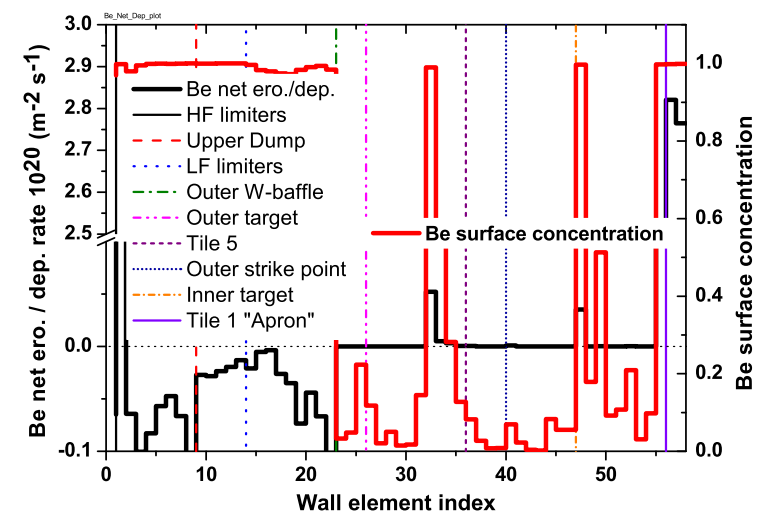

Fig. 2.

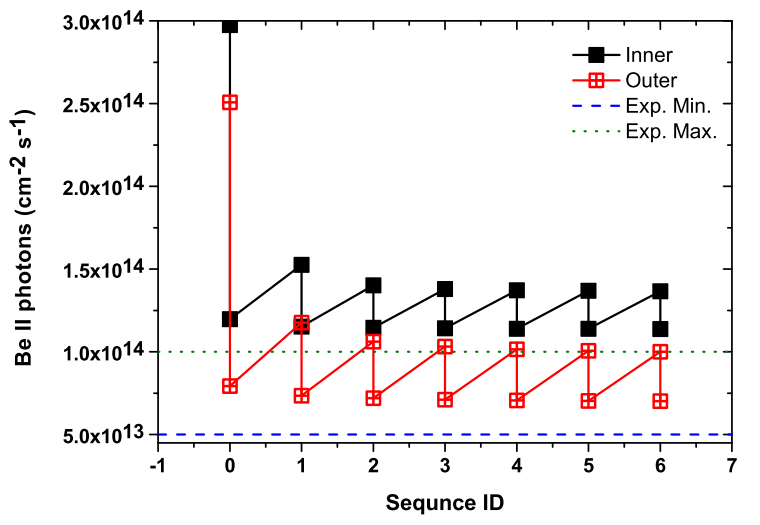

Fig. 3. 


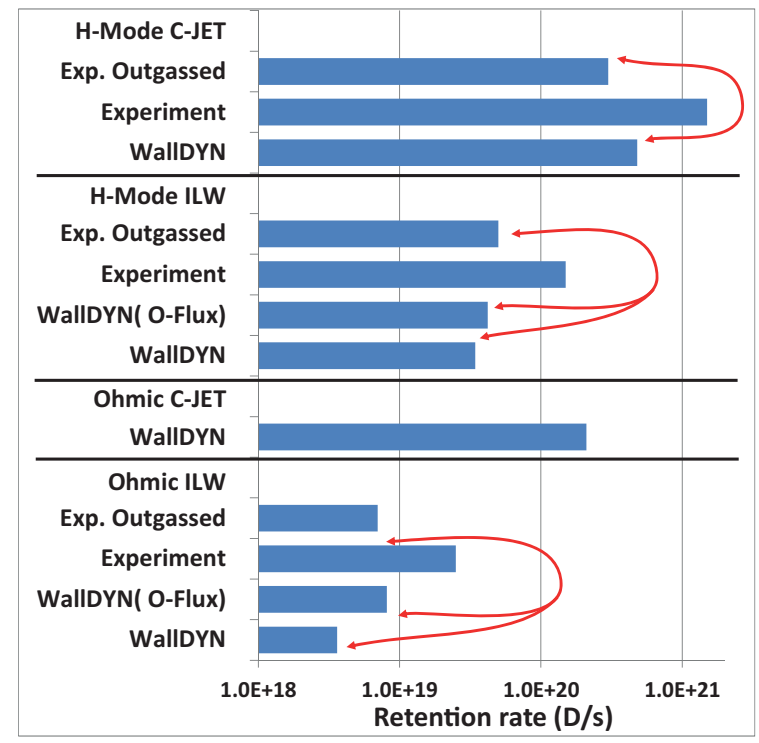

Fig. 4.

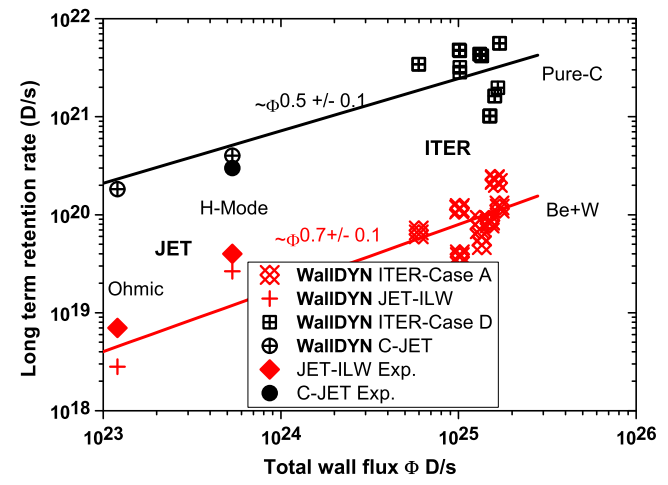

Fig. 5. 


\section{References}

[1] J. Roth, E. Tsitrone, A. Loarte, T. Loarer, et. Al., J. Nucl. Mater. 390-391 (2009) 1.

[2] K. Schmid, M. Reinelt, K. Krieger, J. Nucl. Mater. 415 (2011) 284.

[3] K. Schmid, J. Nucl. Mater. 438 (2013) 484.

[4] W. Möller, W. E. und J. P. Biersack, Computer Physics Communications 51 No. 8 (1988) 355.

[5] A. Mutzke, R. Schneider, W. Eckstein, R. Dohmen, Max-Planck-Institut für Plasmaphysik IPP-Report 12/8.

[6] P. Stangeby, J. D. Elder, J. Nucl. Mater. 196-198 (1992) 258.

[7] G. D. Temmerman, M. Baldwin, R. Doerner, D. Nishijima, K. Schmid, Nucl. Fusion 48 (2008) 075008.

[8] G. D. Temmerman, R. Doerner, Nucl. Fusion 49 (2009) 042002.

[9] G. D. Temmerman, R. Doerner, J. Nucl. Mater. 389 (2009) 479.

[10] V. Philipps, P. Mertens, G. Matthews, H. Maier, JET EFDA contributors, Fusion Engineering and Design 85 (2010) 1581.

[11] K. Krieger, S. Brezinsek, M. Reinelt, S. Lisgo, J. Coenen, S. Jachmich, S. Marsen, A. Meigs, G. van Rooij, M. Stamp, O. van Hoey, D. Ivanova, T. Loarer, V. Philipps, JET EFDA contributors, J. Nucl. Mater. 438 (2013) 262 .

[12] A. Widdowson, E. Alves, C. F. Ayres, A. Baron-Wiechec, S. Brezinsek, N. Catarino, J. P. Coad, K. Heinola, J. Likonen, G. F. Matthews, M. Mayer, M. Rubel, JET EFDA Contributors, Phys. Scr. T159 (2014) 014010.

[13] J. P. Coad, E. Alves, N. P. Barradas, A. Baron-Wiechec, N. Catarino, K. Heinola, J. Likonen, M. Mayer, G. F. Matthews, P. Petersson, A. Widdowson, JET EFDA Contributors, Phys. Scr. T159 (2014) 014012.

[14] S. Brezinsek, T. Loarer, V. Philipps, H. Esser, S. Grünhagen, R. Smith, R. Felton, J. Banks, P. Belo, A. Boboc, J. Bucalossi, M. Clever, J. Coenen, I. Coffey, S.Devaux, D. Douai, M. Freisinger, D. Frigione, M.Groth, A. Huber, J. Hobirk, S. Jachmich, S. Knipe, K. Krieger, U. Kruezi, S. Marsen, G. Matthews, A. Meigs, F.Nave, I. Nunes, R.Neu, J. Roth, M. Stamp, S. Vartanian, U. S. JET EFDA contributors, Nucl. Fusion 53 (2013) 083023.

[15] H. P. Summers, Atomic Data and Analysis Structure Users Manual, Rep. IR(94) 06, JET Joint Undertaking, 1994.

[16] S. Lisgo, P. Stangeby, J. Elder, J. Boedo, B. Bray, N. Brooks, M. Fenstermacher, M. Groth, D. Reiter, D. Rudakov, J. Watkins, W. West, D. Whyte, J. Nucl. Mater. 337 (2005) 256. 
[17] S. Lisgo, A. Kukushkin, R. Pitts, D. Reiter, J. Nucl. Mater. 438 (2013) 580.

[18] R. Schneider, X. Bonnin, K. Borrass, D. P. Coster, Contrib. Plasma Phys. 46 (2006) 191.

[19] D. Borodin, S. Brezinsek, J. Miettunen, M. Stamp, A. Kirschner, C. Björkas, M. Groth, S. Marsen, C. Silva, S. W. Lisgo, D. Matveev, M. Airila, V. Philipps, JET EFDA Contributors, Phys. Scr. T159 (2014) 014057.

[20] S. Brezinsek, S. Jachmich, M. Stamp, A. Meigs, J. Coenen, K. Krieger, C. Giroud, M. Groth, V. Philipps, S. Grünhagen, R. Smith, G. van Rooij, D. Ivanova, G. Matthews, JET EFDA contributors, Submitted to Nuclear Fusion.

[21] V. Philipps, T. Loarer, H. Esser, S. Vartanian, U. Kruezi, S. Brezinsek, G. Matthews, JET EFDA contributors, J. Nucl. Mater. 438 (2013) 1067. 